\title{
UN PROYECTO PARA LA CONSERVACIÓN DE LA ENTOMOFAUNA SAPROXÍLICA EN ECOSISTEMAS MEDITERRÁNEOS
}

\section{E. Micó ${ }^{1}$, M.A. Marcos García, M.A. Alonso ${ }^{1}$, C. Pérez- Bañón ${ }^{1}$, A. Padilla ${ }^{2}$ y T. Jover ${ }^{1}$}

1 CIBIO, Universidad de AliCANTE

2 Instituto Universitario de Geografía, Universidad de Alicante e.mico@ua.es

\section{INTRODUCCION}

La historia del uso de los bosques en Europa se encuentra bien documentada; los bosques apenas habían alcanzado su mayor extensión postglacial, cuando comenzaron a ser explotados por el hombre con la llegada del Neolítico hace 7000 años. A lo largo de los últimos cinco milenios, la cobertura forestal ha disminuído drásticamente y la estructura y composición de los fragmentos restantes, se ha visto profundamente alterada (THIRGOOD, 1989). Revisando la pérdida progresiva de biodiversidad animal asociada a los bosques por el "uso y abuso" forestal, Groove (2002) define la explotación forestal en Europa como "a 5000-year experiment in unsustainable management" = un experimento de 5000 años de uso no sostenible.
Tanto los árboles muertos (caídos o en pie) como los grandes árboles provistos de numerosas oquedades, son elementos estructurales de los bosques que representan el recurso dominante de materia orgánica, constituyendo a su vez un microhábitat de características muy peculiares. A las especies que dependen, durante al menos parte de su ciclo vital, de la madera o sus restos, así como de los hongos asociados a dicho medio, se las denomina saproxílicas (SPEIGHT, 1989). El papel ecológico de la madera muerta se presenta de diversas formas en los ecosistemas, mejorando la eficiencia hidrogeológica de las montañas, favoreciendo la formación de humus, y contribuyendo al mantenimiento de la biodiversidad dando cobijo y alimento a las comunidades de hongos, invertebrados y vertebrados. 
Además de su importancia ecológica, actuando en la degradación e incorporación de nutrientes al ecosistema, son los invertebrados saproxílicos los que constituyen la mayor parte de la biodiversidad en los ecosistemas terrestres (SChlaghamersKy, 2003), siendo los Insectos Dípteros y Coleópteros los grupos mejor representados entre la fauna saproxílica. Los escarabajos y moscas saproxílicas vienen siendo objeto de numerosos estudios en Europa durante los pasados 10-20 años, debido a que constituyen el mayor componente de la biodiversidad de sus bosques. Sin embargo, el porcentaje de especies saproxílicas raras y amenazadas en Europa es muy elevado y las extinciones regionales son cada vez más frecuentes (GEISER, 1983; SHIRT, 1987).

La madera muerta es una pieza clave para la conservación de la biodiversidad debido a que constituye un componente funcional que asegura el almacenamiento de nutrientes del ecosistema. A su vez, presenta especial importancia en el mantenimiento adecuado de la cadena alimenticia actuando como microhábitat indispensable para la supervivencia de numerosas especies de Briofitos, Roedores, Murciélagos, Aves e Insectos. Los árboles marcescentes ofrecen muy distintos microhábitat caracterizados por la elevada estabilidad de sus parámetros físicos (temperatura, humedad, luz) y por la continua disponibilidad de nutrientes. Por este motivo, miles de especies conforman la biodiversidad asociada a los árboles muertos y a sus oquedades. Sin embargo, a pesar de constituir un componente esencial de los bosques, los árboles muertos y sus restos están ausentes en la mayoría de las masas forestales explotadas por el hombre. Este gap lleva asociado una pérdida de microhábitat y de recurso alimenticio, lo que conlleva severas amenazas a la rica y original biodiversidad de las comunidades saproxílicas de hongos e invertebrados.

Las políticas medioambientales seguidas en la actualidad en la Comunidad Europea, están dirigidas a conservar y gestionar el medio en pro de la conservación de la biodiversidad. En este sentido, y en especial durante los últimos 15 años, se tiende hacia la búsqueda de nuevas vías para un manejo sustentable de las masas forestales. La importancia de las especies saproxílicas ha sido reconocida a nivel Europeo (COUNCIL OF EUROPE, 1988) y son numerosos los proyectos LIFE-NATURE

(http://europa.eu.int/comm/environment/life) financiados por los fondos Europeos, que apuestan por el estudio de la fauna saproxílica y la búsqueda de soluciones ante la acuciante pérdida de biodiversidad de los mismos (SANDSTRÖM, 2003). Sin embargo, las iniciativas de este tipo se han centrado hasta ahora en los bosques boreales y en aquellos de zonas templadas centroeuropeas, mientras que apenas se conocen datos aislados sobre la biodiversidad entomológica asociada a la madera en bosque mediterráneo.

La originalidad del proyecto "Evaluación del estado de conservación del bosque mediterráneo: bases para la gestión de la entomofauna saproxílica asociada" que aquí se presenta, reside en que se trata de una investigación dirigida a evaluar la biodiversidad saproxílica del bosque mediterráneo con el objeto de proponer medidas para su gestión y conservación. Uno de los aspectos, tan novedoso como necesario, de esta investigación será el de proporcionar un estudio comparable con los llevados a cabo en otros países comunitarios que nos permita establecer Índices de Calidad del medio Saproxílico aplicables a nuestros bosques. Por su peculiar enclave biogeográfico, donde confluyen vías de dispersión de especies africanas y centroeuropeas, los bosques de la península Ibérica albergan una rica entomofauna en la que destacan componentes europeos y africanos que encuentran aquí su límite de distribución. En este sentido, desde la última glaciación, son muchas las especies saproxílicas que encontraron refugio concretamente en el sur de la península Ibérica, Italia, los Balcanes y Próximo Oriente (Aberlenc eT Al., 2003). El estudio preliminar de esta fauna saproxílica y sobretodo de sus requerimientos biológicos es sin duda necesario para proponer medidas de gestión dirigidas a conservar la biodiversidad forestal en la región Mediterránea. Muchas de las especies de Dípteros más raras y amenazadas dependen de los microhábitat que ofrecen las 
oquedades de los árboles, mientras que otras especies asociadas a la madera muerta, presentan una amplitud ecológica mucho mayor (SPEIGHT, 1989), por lo que las acciones para proteger a la fauna han de ser distintas en cada caso. Por este motivo, conocer las biologías particulares de los grupos indicadores constituye un paso previo y necesario a la acción para su adecuada protección. Por último, cabe destacar que cada uno de los aspectos propuestos en este proyecto, constituyen en sí mismo una contribución al avance científico dirigido al conocimiento del bosque mediterráneo y aplicado a la mejora de la gestión de nuestro patrimonio natural y conservación de la biodiversidad.

\section{OBJETIVOS DEL PROYECTO}

El objetivo general es evaluar la biodiversidad de la entomofauna saproxílica propia de los ecosistemas de bosque mediterráneo, analizando de forma comparativa su relación con los distintos tipos de formaciones arbóreas y con el microhábitat (en referencia al nicho ecológico explotado). Se pretende así mismo traducir los resultados acerca de la distribución y requerimientos biológicos de la mencionada entomofauna indicadora en la propuesta de medidas para conservación de la biodiversidad saproxílica y la gestión de su hábitat. Con el fin de desarrollar la investigación propuesta se han seleccionado distintos grupos entomológicos pertenecientes a los Dípteros y Coleópteros en función de su idoneidad como grupos indicadores y por sus distintos requerimientos biológicos.

Para la consecución de los objetivos planteados hemos seleccionado tres medios o mesohábitat que caracterizan a las principales formaciones arbóreas presentes en el área mediterránea:

- Formaciones arbóreas dominadas por especies esclerófilas [Alcornoque (Quercus suber)].

- Formaciones arbóreas dominadas por especies marcescentes [Robeldal (Quercus pyrenaica)-Quejigal (Quercus faginea)].

- Formaciones riparias [Fresnedas (Fraxinus angustifolia)].
Los objetivos concretos planteados son los siguientes:

1. Análisis comparativo de la diversidad de la entomofauna saproxílica de las tres formaciones arbóreas (mesohábitat) seleccionadas. Los resultados de este primer objetivo nos permitirán elaborar las primeras listas de entomofauna ibérica saproxílica asociadas a bosque mediterráneo.

2. Análisis de la diversidad de la entomofauna saproxílica asociada a los distintos microhábitat que ofrecen las distintas formaciones arbóreas (oquedades de los árboles, troncos en pie, troncos caídos, ramas y tocones, etc...). Asimismo, se analizará la composición físico-química de los diferentes microhábitat mencionados con objeto de caracterizar de forma más precisa cada uno de los microambientes que explota la entomofauna. Los resultados de este objetivo se verán plasmados en una base de datos que clasificará a las distintas especies en función de su requerimiento biológico. La distribución de los nichos ecológicos y su entomofauna asociada será también cartografiada en cada una de las parcelas.

3. Valoración del estado actual de los ecosistemas de bosque mediterráneo (escala de paisaje o macrohábitat) y elaboración de propuestas para la conservación de la entomofauna saproxílica.

\section{PARTICIPANTES Y FINANCIACIÓN DEL PROYECTO}

El equipo está constituido por seis investigadores pertenecientes a dos Institutos Universitarios de Investigación de la Universidad de Alicante, el CIBIO (Centro Iberoamericano de la biodiversidad) y el Instituto de Geografía. Desde el reciente nacimiento de la ciencia de la Biología de la Conservación como respuesta a la actual crisis de la diversidad biológica, se ha puesto de manifiesto la necesidad de abordar los problemas desde un enfoque multidisplinar como única vía para aportar soluciones factibles. En nuestro caso concreto, los resultados de las colaboraciones entre entomólogos, botánicos y geógrafos para abordar estudios de biodiversidad y conservación del medio corroboran la impor- 
tancia de conjugar los conocimientos y metodologías que aportan las distintas disciplinas.

A este grupo se suma la colaboración científica de investigadores extranjeros de reconocido prestigio en la conservación y estudio de la entomofauna saproxílica como el Dr. G. Rotheray (National Museum of Scontland), el Dr. G. Hancock (University of Glasgow) y el Dr. M. Speight (Research Branch, National Parks \& Wildlife, Ireland).

Esta iniciativa del CIBIO se encuentra respaldada por la Generalitat Valenciana (GV04A576) a la vez que cuenta con la cofinanciación de los proyectos "Biodiversidad Entomológica del parque Nacional de Cabañeros: Caracterización mediante un Sistema de Información Geográfica y propuestas para el Plan de Uso y Gestión” subvencionado por el Ministerio de Medioambiente bajo la dirección de la Dra. M. Ángeles Marcos García y "La entomofauna saproxilica como indicador del estado de conservación el bosque Mediterráneo: Bases para la conservación de su biodiversidad" subvencionado por la Universidad de Alicante (GRE04-13) bajo la dirección de la Dra. Estefanía Micó Balaguer.

\section{EL ÁREA DE ESTUDIO}

Como base para el desarrollo de este proyecto de investigación se ha seleccionado el Parque Nacional de Cabañeros como representante de ecosistema mediterráneo constituido típicamente por un diverso mosaico de distintas unidades arbóreas, arbustivas y herbáceas con distintos grados evolutivos y estructuras.

El Parque Nacional de Cabañeros tiene una extensión de $41.804 \mathrm{Ha}$ y se encuentra situado entre las provincias de Ciudad Real y Toledo. Su declaración, primero como Parque Natural en 1988 y más tarde como Parque Nacional a finales de 1995 , se debió en parte a su singular forma de propiedad mantenida durante seis siglos que ha compatibilizado el uso de sus recursos naturales con la conservación de su flora y fauna y que ha dado lugar así mismo a un paisaje singular.

La presencia del hombre en el área de estudio desde los primeros asentamientos del Paleolítico ha ido transformado el paisaje, principalmente en lo que se refiere a abastecimiento de madera, leña y carbón vegetal. Sin embargo, a partir del siglo XIII la regulación de la explotación fue tan estricta que permitió un sistema de uso y explotación similar al de un espacio natural protegido. De esta manera, la selección de un área de estudio en la que se han mantenido especies forestales conservadas a lo largo del tiempo, nos permitirá en un futuro sentar las bases de comparación de la biodiversidad saproxílica con otras áreas forestales mediterráneas que sufren un manejo más intenso. Las bases sentadas con este trabajo permitirán el desarrollo de esta línea de investigación profundizando en aspectos como conocer el efecto de la fragmentación del hábitat en la biodiversidad saproxílica, el efecto del tamaño y la forma de la masa forestal en la pervivencia de las especies, el efecto del aclareo (natural $\mathrm{y}$ artificial) en la entomofauna asociada, etc.

Una de las acciones previas para el desarrollo de esta investigación ha sido caracterizar la vegetación de los puntos de muestreo, geoposicionarlos y realizar la cartografía. A continuación se detallan las características de cada uno de los puntos de muestreo o mesohábitat seleccionados:

\section{"Melojar de Viñuelas"}

El punto del muestreo del melojar se sitúa en el sector central del parque, al norte de la Raña de la Viñuela (X: 371.000; Y: 4.359.000) (Figura 1) y pertenece al piso bioclimático mesomediterráneo subhúmedo, aunque los melojares son etapas climáticas del piso supramediterráneo húmedo. El caso del melojar de Viñuelas al igual que otros melojares del Parque responden a unas condiciones topográficas favorables. En muchas ocasiones estos melojares están entremezclados con el quejigo (Quercus broteroi) que también busca estas zonas más húmedas y frías. Es por esta razón por lo cual no es extraño encontrar en los valles del Parque bosques mixtos de Melojos, Quejigos y Fresnos.

\section{"Alcornocall de Gargantilla"}

El alcornocal elegido se ubica en la zona de Gargantilla, situada en el sector occidental del parque, concretamente en la vertiente septentrional del 


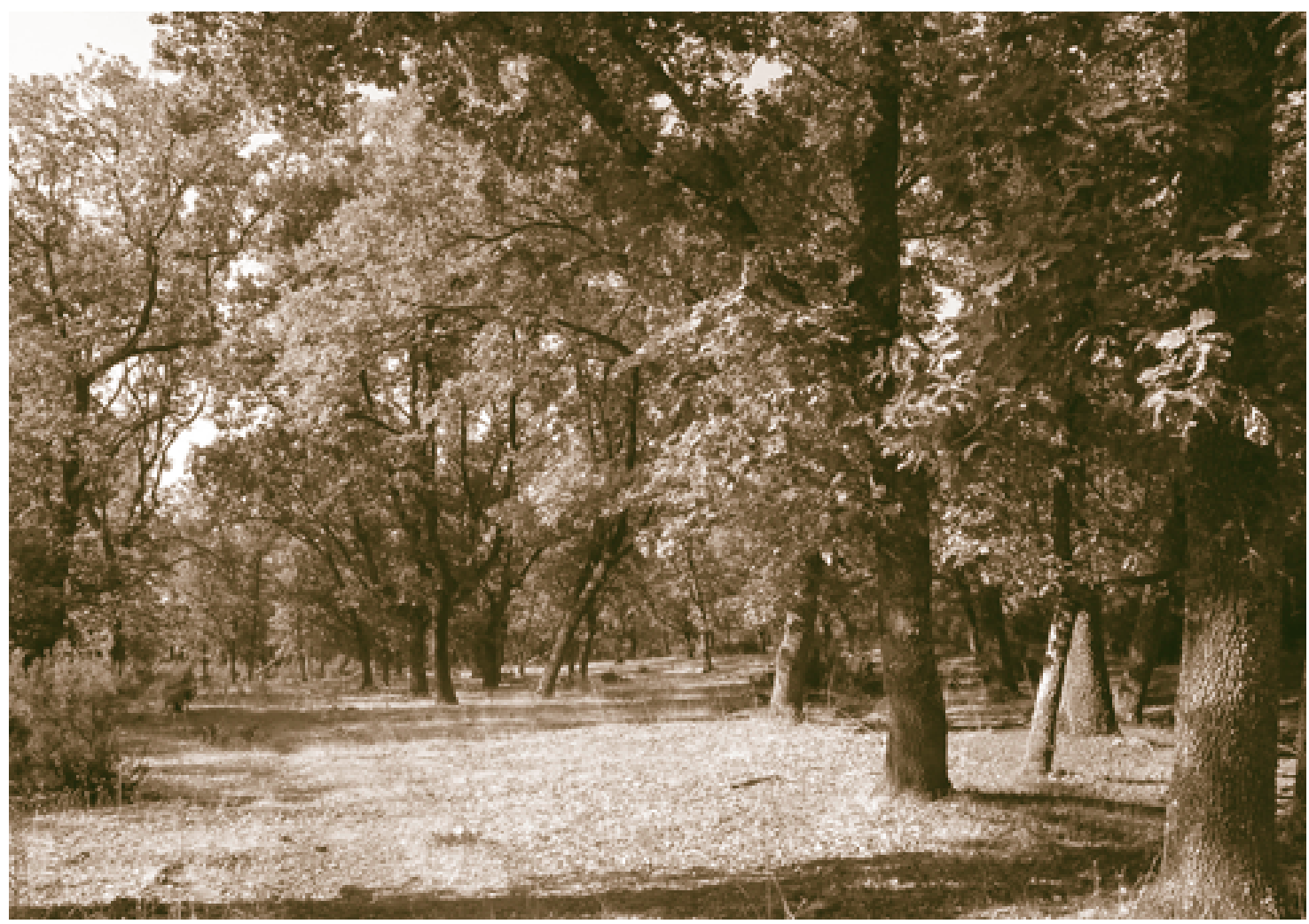

Figura 1. Melojar (Quercus pyrenaica) del Valle de la Viñuela.

cerro de Utrera, en la cabecera del Arroyo de Gargantilla (X: 362.000; Y: 4.367.000) (Figura 2).

Se trata de un bosque más o menos abierto que ocupa situaciones topográficas abruptas sobre suelos muy pobres que no tienen aprovechamiento agropecuario, preferentemente en las solanas con menor riesgo de fuertes heladas. Este bosque suele estar acompañado por especies como Sanguisorba agrimonioides y Peonia broteroi. El mayor aprovechamiento del alcornocal ha sido, hasta la creación de la figura de Parque Nacional, la corteza del propio alcornoque para la producción de corcho.

\section{"Valle de Canallejas"}

Este punto de muestreo se localiza en el sector oriental del parque, concretamente en el Arroyo de las Canalejas, aguas arriba de la confluencia con el Arroyo de Cebea en el paraje denominado La Sarten (X: 377.000 y 378.000; Y: 4.358.000) (Figura 3).
Los Quejigares de Quercus faginea subsp. broteroi en el Parque Natural de Cabañeros no son comunidades climácicas sino que aprovechan fondos de valle, donde son favorecidos por la profundidad de suelo y la humedad edáfica (Vaquero de la Cruz, 1996), ésta es la razón por la cual es usual que convivan con los Fresnos (Fraxinus angustifolia).

El quejigar-fresneda elegido se sitúa en el valle de Canalejas. Este valle está atravesado por un rambla, los quejigos se sitúan en la ladera con una pendiente suave de un $10 \%$ a medida que los quejigos se acercan al arroyo, se entremezclan con fresnos (Fraxinus angustifolia) que a su vez también se alejan un poco del borde del arroyo. El sotobosque es muy pobre en especies y lo más significativo es la vegetación acuática que se instala en los regueros que cruzan la ladera dirección al arroyo. Los restos de vegetación que se observan en el sotobosque corresponden a las etapas de degradación de la comunidad climácica que es el Sanguisorbo-Querceto suberis sigmetum. 


\section{Cuadernos de Biodiversidad}

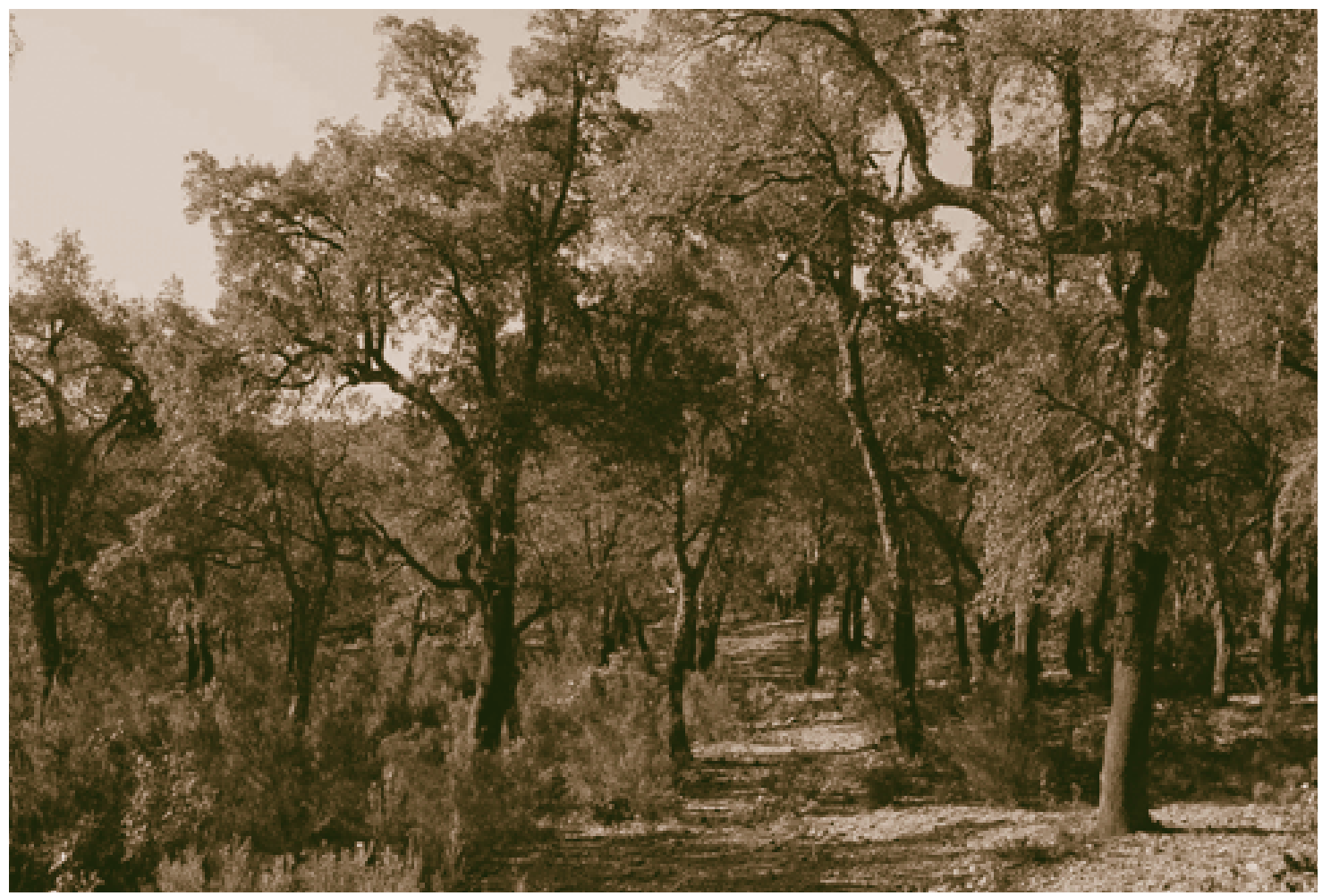

Figura 2. Alcornocal (Quercus suber) de Gargantilla.

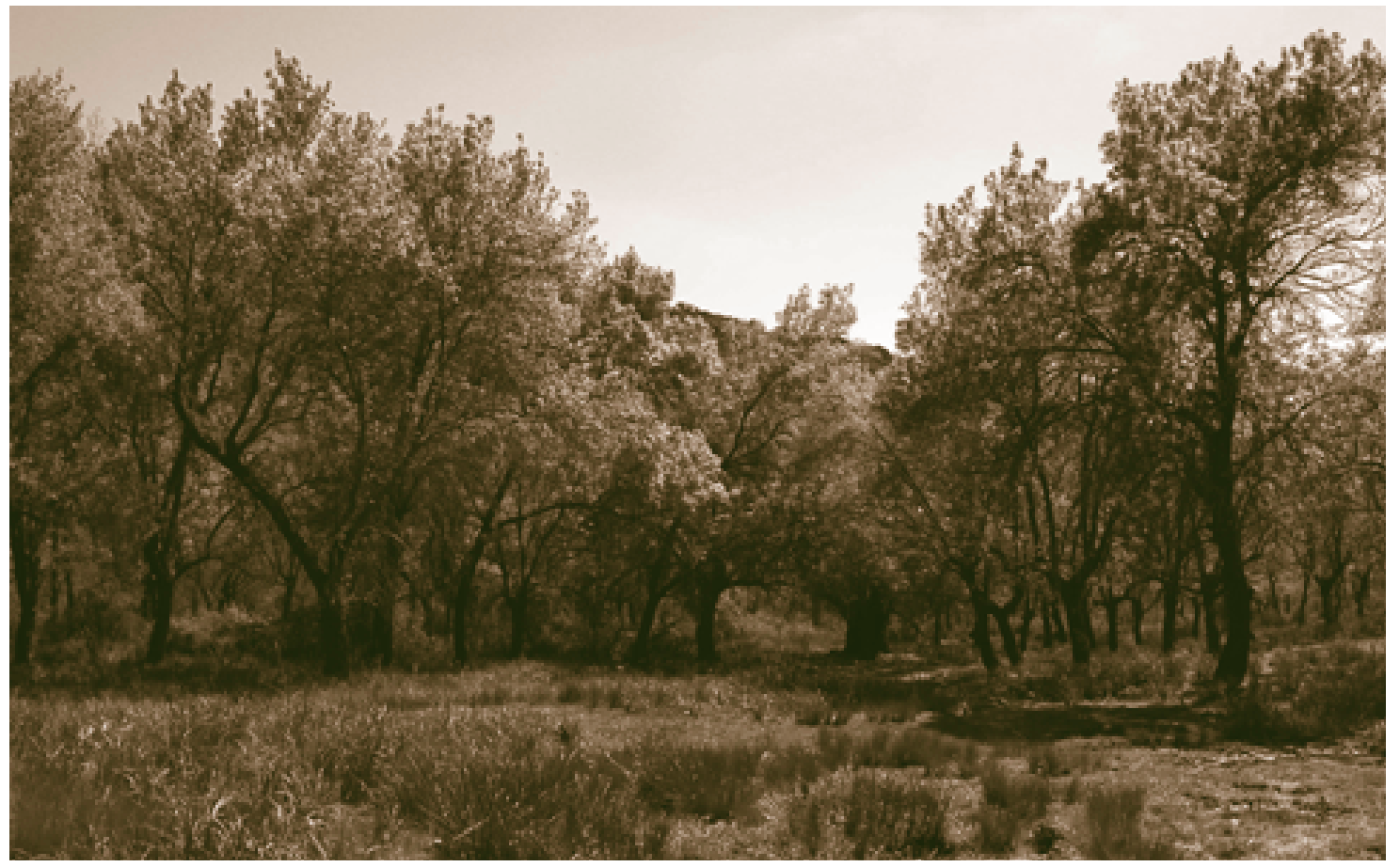

Figura 3. Quejigar (Quercus faginea broteroi) del Valle de Canalejas. 


\section{"Fresneda de Gargantilla"}

Los fresnos (Fraxinus angustifolia) de la fresneda del Arroyo de la Gargantilla, que discurre paralelo al río Estena, se localiza en la mitad septentrional del parque (X: 365.000; Y: 4.367.000) (Figura 4).

La serie del Fresno (Ficario-Faxineto angustifoliae sigmetum) corresponde en su estapa madura, como en otras series riparias lusiextremadurense la orla espinosa del bosque es un zarzal con clemátides (Clemati campaniflorae-Rubetum ulmifolii).

\section{RESULTADOS PRELIMINARES}

Durante esta primera fase del proyecto se aborda la puesta en marcha y desarrollo de los dos primeros objetivos: "Análisis comparativo de la diversidad de la entomofauna saproxilica de las distintas formaciones arbóreas" y "Análisis de la

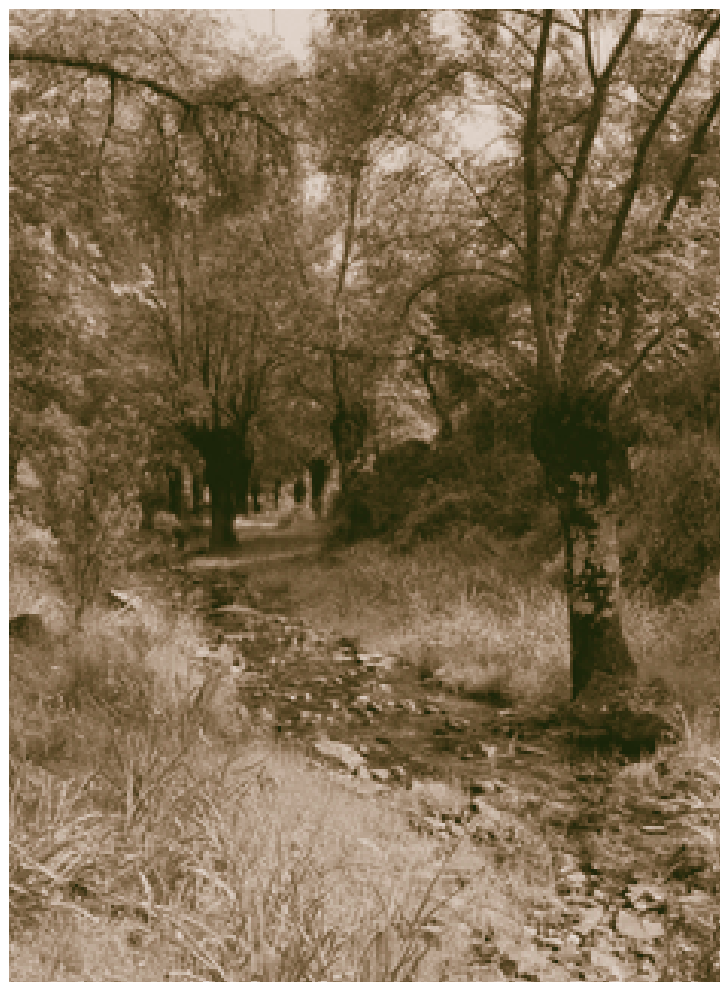

Figura 4. Fresneda (Fraxinus angustifolia) de Gargantilla. diversidad de la entomofauna saproxílica asociada a los distintos microhábitat que ofrecen los árboles".

\section{Estudio comparativo de la biodiversidad de ento-} mofauna saproxílica de las distintas formaciones arbóreas

Para desarrollar este objetivo se están realizando muestreos tanto de tipo directo como indirecto en cada uno de los mesohábitat seleccionados. El muestreo indirecto se ha llevado a cabo mediante la utilización de trampas de intercepción. Se ha dispuesto, en cada medio, un total de 7 trampas de intercepción (Windowtraps) colgadas de los árboles a 1,5-2,5 $\mathrm{m}$ del suelo (Figura 5). Dichas trampas están destinadas al muestreo de los Coleópteros voladores que acuden a los troncos de los árboles vivos y las colectas son efectuadas con una periodicidad mensual.

Los resultados preliminares son los siguientes:

La utilización de trampas de intercepción (Windowtraps) en los distintos mesohábitat se ha mostrado

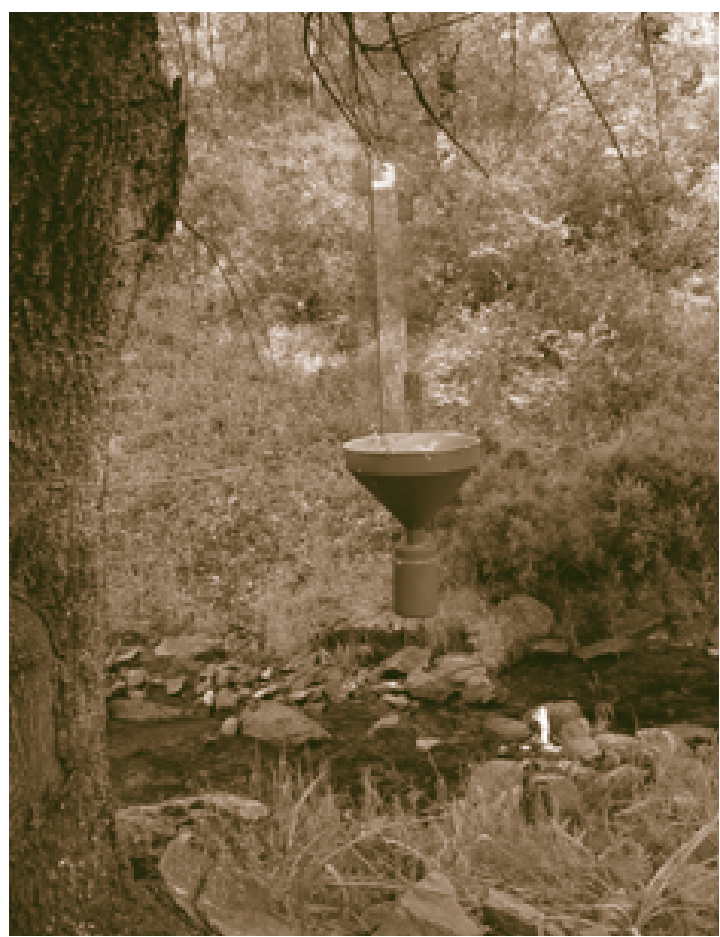

Figura 5. Detalle de las trampas de intercepción de vuelo ("Window traps") utilizadas en el muestreo indirecto de Coleópteros saproxilicos. 
efectiva en la colecta de Coleópteros saproxílicos asociados, habiéndose colectado un total de 1.728 individuos. Las diversidades más elevadas, tanto a nivel familia como en riqueza de individuos se han obtenido en las formaciones arbóreas dominadas por especies marcescentes de Quercus faginea y Quercus pyrenaica. Sin embargo, estos resultados se corresponden únicamente al periodo de muestreo primaveral, datos que deberán ser completados al final del presente año con los muestreos otoñales.

\section{Análisis de la diversidad de la entomofauna saproxílica asociada a los distintos microhábitat que ofrecen los árboles}

Si bien existen distintas metodologías para estudiar la fauna saproxílica, asociada a un ecosistema, hay que destacar que el muestreo directo realizado por especialistas es el que aporta más información. Concretamente, es el muestreo directo de fases larvarias el que nos proporciona la información necesaria sobre los requerimientos biológicos de estos grupos indicadores (Figura 6). El muestreo directo se ha centrado en la colecta de las fases larvarias de los Coleópteros Scarabaeoidea y de los Dípteros Syrphidae (Figura 7) como grupos indicadores de la entomofauna saproxílica. Debido a que son estas fases preimaginales las que se encuentran más estrechamente ligadas al medio saproxílico hemos realizado colectas sistemáticas en los distintos microhábitat que ofrecen las formaciones arbóreas tales como oquedades de troncos, ramas y raíces de árboles vivos así como en los árboles muertos en pie o caídos presentes en los medios seleccionados. Las fases larvarias colectadas en campo se llevaron al laboratorio para su identificación y su posterior cría bajo condiciones de temperatura y humedad controladas.

Las larvas hasta ahora colectadas han sido identificadas, y se ha creado una base de datos en la que se reúne toda la información obtenida en campo sobre el nicho ecológico de cada una de las especies.

Los resultados, referidos al periodo primaveral, han sido los siguientes:
- Dípteros Syrphidae

En total se ha colectado y criado en laboratorio 155 larvas de 8 especies de sírfidos: Callicera aurata (Rossi, 1790), C. spinolae Rondan, 1844, C. maquartii Rondani, 1844, Myathropa florea (Linnaeus, 1758), Ceriana vespiformis (Latreille, 1804), Spilomyia digitata (Rondani, 1865), Myolepta difformis Strobl, 1909 y Mallota dusmeti Andreu, 1926.

Es de destacar que dos de ellas $(M$. dusmeti y $M$. difformis) son especies endémicas de la península ibérica y de escasa presencia en la misma, por lo que estos resultados representan una gran importancia en las estrategias de su conservación.

Los datos de biología larvaria de $M$. dusmeti son especialmente relevantes ya que esta especie ha sido propuesta para ser incluidas en la Lista Roja de especies de insectos amenazadas en la península Ibérica.

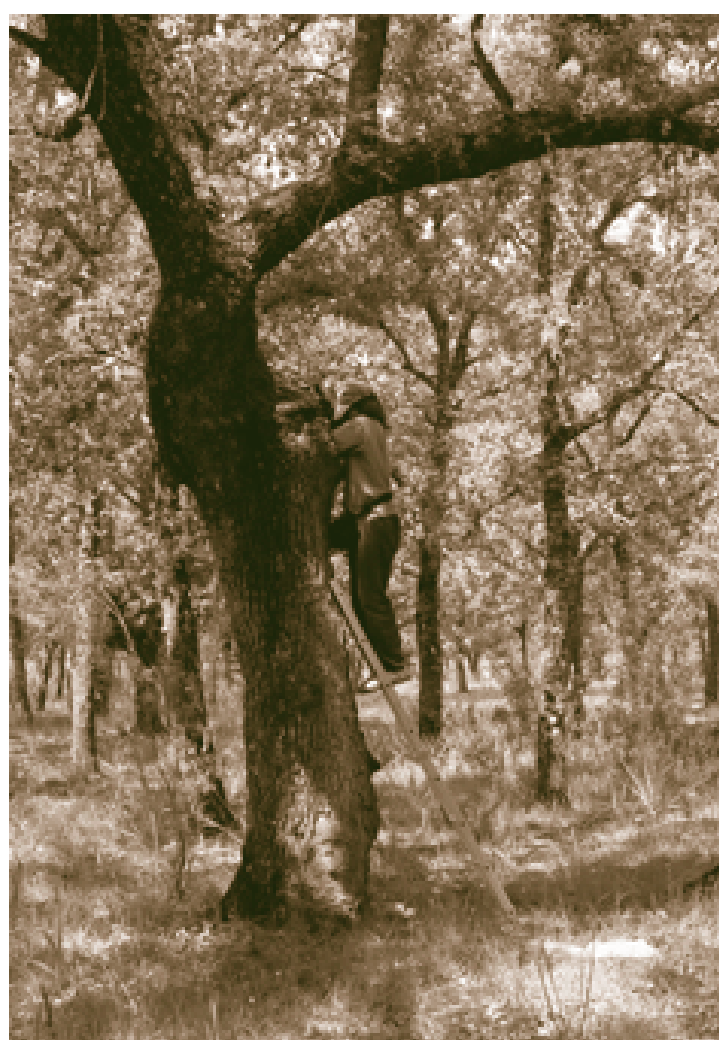

Figura 6. Muestreo directo realizado en las oquedades de los árboles. 


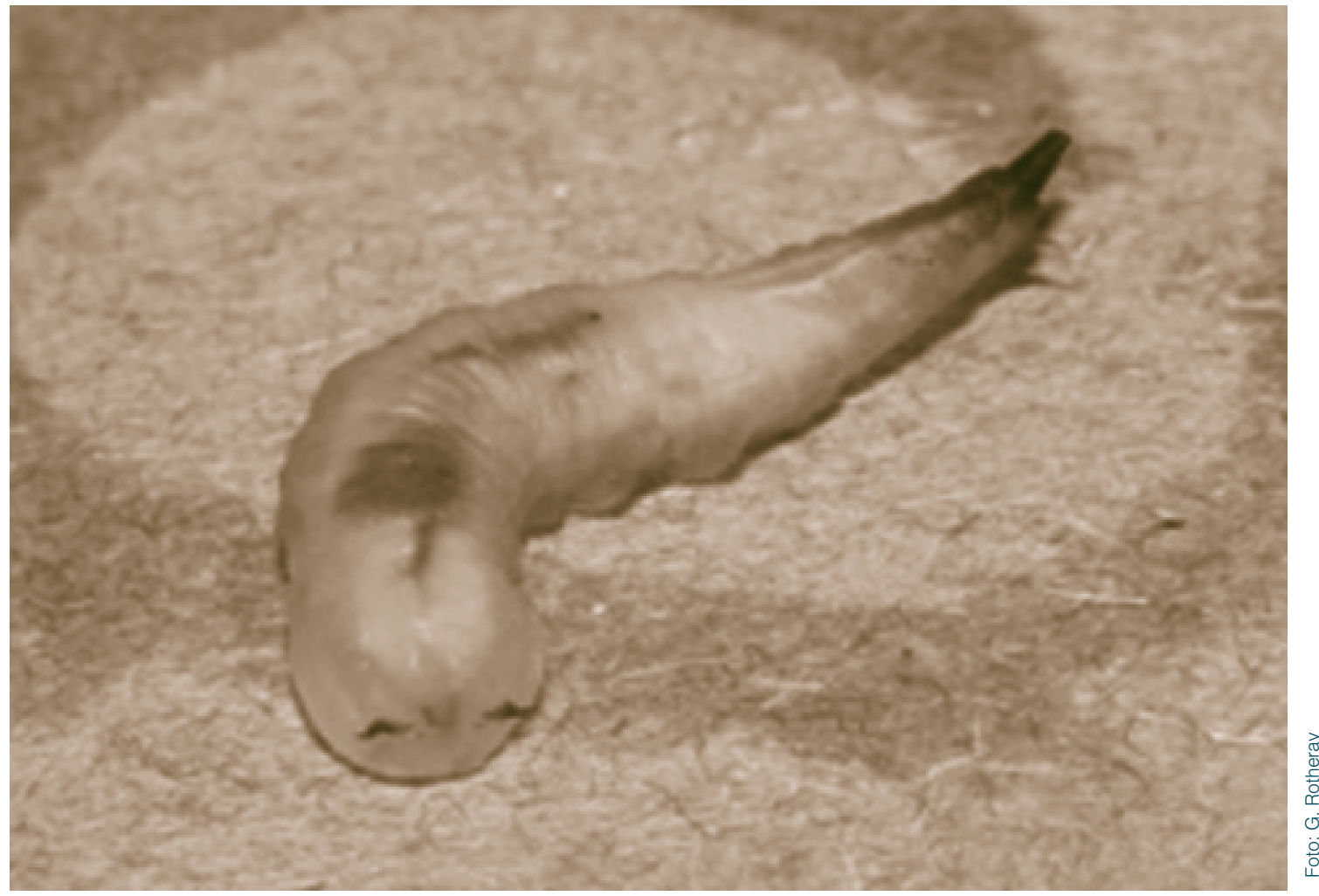

Figura 7. Larva de Callicera spinolae (Diptera: Syrphidae)

Hay que destacar que los datos de cría hasta ahora obtenidos son los primeros que se conocen de estas especies en ecosistemas mediterráneos, ya que hasta el momento, sólo se conocían citas aisladas de ejemplares adultos de dichas especies.

Los datos de biología larvaria de Ceriana vespiformis son los primeros que se conocen sobre la biología larvaria de este género.

Las larvas de todas estas especies se desarrollan en oquedades de cierto tamaño situadas en árboles maduros y conteniendo una gran cantidad de agua retenida en su interior donde se producen procesos de descomposición de materia orgánica vegetal.

\section{- Coleópteros Scarabaeoidea}

Se ha colectado un total de 73 larvas de siete especies de Escarabeidos pertencecientes a las familias Cetoniidae, Dynastidae y Lucanidae: Cetonia auratiformis Curti, Potosia opaca (Fabri- cius), Potosia cuprea brancoi Baraud y Trichius zonatus Gemar (Cetoniidae), Oryctes nasicornis (Linnaeus) (Dynastidae), y Pseudolucanus barbarossa (Fabricius) (Figura 8) y Dorcus parallelepipedus (Linnaeus) (Lucanidae).

La especie mayoritaria encontrada hasta ahora en los distintos medios fue el endemismo ibérico Cetonia auratiformis (Cetoniidae). Hay que destacar que la asociación de las fases larvarias de la mayoría de estas especies a las distintas especies arbóreas supone una novedad para la ciencia.

Las larvas de estas especies se han encontrado principalmente en las distintas oquedades que presentan los árboles vivos de gran tamaño, fundamentalmente en las oquedades del tronco y de las raíces. Dichas oquedades constituyen microhábitat caracterizados por presentar abundante materia orgánica en descomposición como fuente de alimento para las larvas, así como por la estabilidad de las condiciones de humedad y temperatura. 


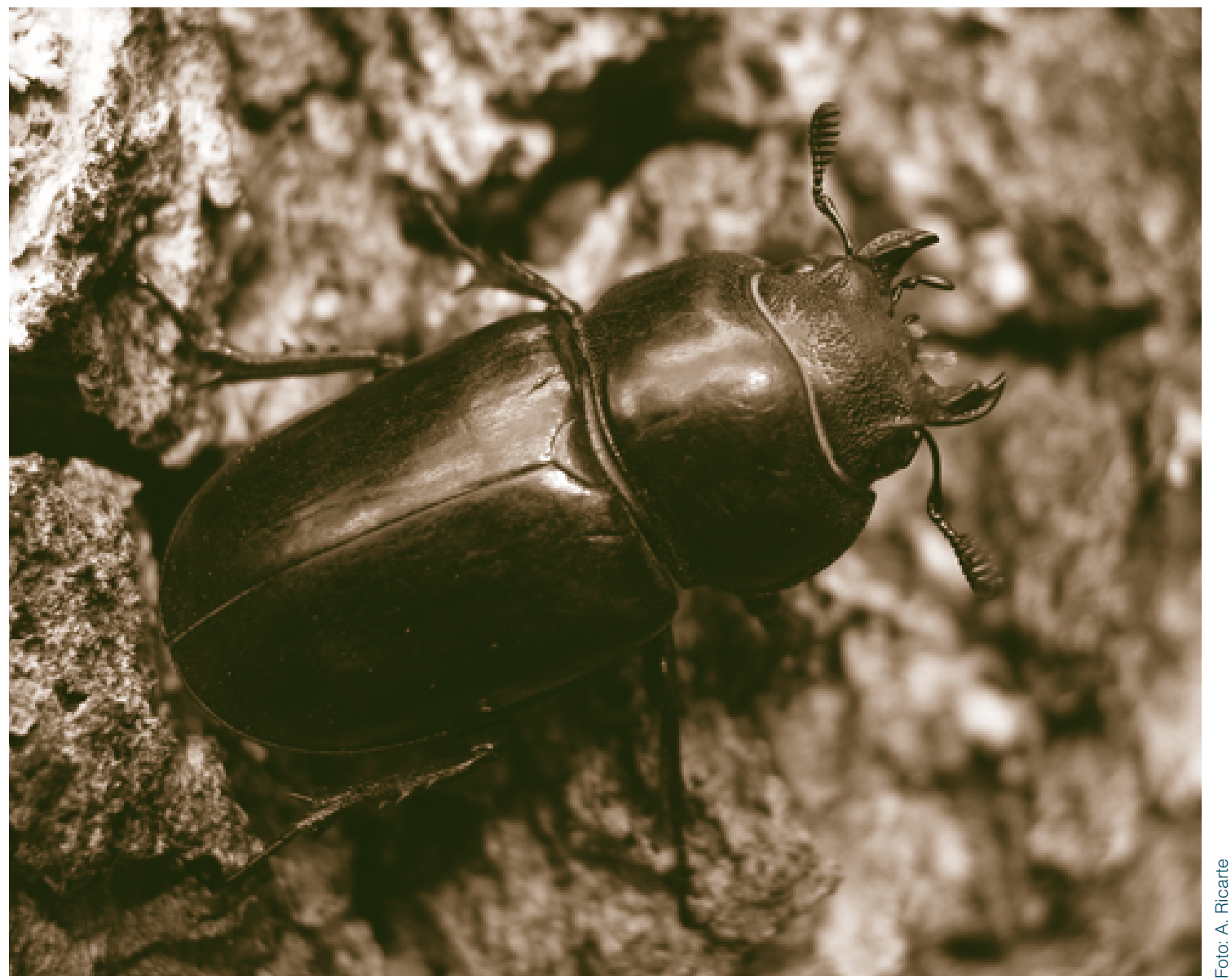

Figura 8. Hembra de Pseudolucanus barbarossa (Scarabaeoidea: Lucanidae).

Hay también que destacar que para los dos grupos indicadores aquí estudiados (Sírfidos y Escarabeidos) es la especie Fraxinus angustifolia la que ofrece un mayor número de nichos ecológicos para los insectos saproxílicos. El carácter más blando de su madera la convierte en una especie de crecimiento más rápido en la que abundan las grandes oquedades tanto en su tronco como en sus raíces.

\section{PERSPECTIVAS}

El interés y originalidad de los objetivos planteados estriba en que es la primera vez que se aborda en el bosque mediterráneo un estudio en el que se utilizan diversos grupos entomológicos para reflejar la biodiversidad saproxílica.
Los datos obtenidos podrán ser aplicados a la selección de las zonas de mayor interés para la entomofauna saproxílica de este Parque Nacional y en la propuesta de medidas de gestión del hábitat. A su vez, supondrá la base para elaborar Indices de Calidad del medio saproxílico (SQI), que hasta ahora únicamente han sido utilizados en ciertos países pioneros (FOWLES ET AL. 1999).

Tras la consecución de los primeros 6 meses de proyecto, los resultados preliminares que aquí se presentan sobre la entomofauna saproxílica en el Parque Nacional de Cabañeros revelan interesantes novedades sobre la biología de numerosas especies indicadoras y su asociación con especies arbóreas del bosque mediterráneo a la vez que sientan las bases para la consecución de los distintos objetivos previstos. 


\section{AGRADECIMIENTOS}

Queremos agradecer al Parque Nacional de Cabañeros y en su nombre al director del Parque D. José Jiménez por su inestimable ayuda en el desarrollo de nuestra investigación.

\section{REFERENCIAS CITADAS}

ABERLENC, H.P, CURLETTI, G., DULTO, M, TASSI, F. 2003. Eupotosia mirifica, joyau menacé du patrimoinie naturel Européen (Coleoptera, Cetoniidae). (En: Dead wood: a key to biodiversity. Proceedings of the International Symposium 29-31 May 2003. Eds: F. Mason, G. Nardi, M. Tisato. Mantova Italy, 99 pp.).

FOWLES, A.P., ALEXANDER, K.N.A. \& KEY, R.S. 1999. The Saproxylic Quality Index: evaluating wooded habitats for the conservation of dead-wood Coleoptera. The Coleopterist, 8: 121141.

GEISER, R. 1983. Red list of threatened xylobiont beetle families (Coleoptera) in Austria [in German] ed. J. Gepp, pp. 131-137. Wien, Austria: Österr. Bundesminist. Gesundh. Umweltschutz.
GROOVE, S.J. 2002. Saproxylic insect ecology and the sustainable management of forests. Annu. Rev. Ecol. Syst. 33: 1-23.

SANDSTRÖM, E. 2003. Dead wood: objective, results and life-projects in swedish forestry. (En: Dead wood: a key to biodiversity. Proceedings of the International Symposium 29-31 May 2003. Eds: F. Mason, G. Nardi, M. Tisato. Mantova Italy, 99 pp.).

SCHLAGHAMERSKY, D.J. 2003. Saproxylic invertebrate of foodplains, a particulary endangered component of biodiversity. (En: Dead wood: a key to biodiversity. Proceedings of the International Symposium 29-31 May 2003. Eds: F. Mason, G. Nardi, M. Tisato. Mantova Italy, 99 pp.).

SHIRT, B.D. 1987. British Red Data Books: 2. Insects. Peterborough, UK: Nat. Conserv. Counc.

SPEIGHT, M.C.D. 1989. Saproxylics invertebrates and their conservation. Council of Europe, Strasbourg, $66 \mathrm{pp}$.

THIRGOOD, J.V. 1989. Man's impact on the forest of Europe. J. World For. Resour. Manage. 4: 127-167. 